Fizika Nizkikh Temperatur, 1998, v. 24, No 10, p. 984-991

\title{
Electron-impact desorption of metastable particles from CO films
}

\author{
H. Shi, P. Cloutier, and L. Sanche \\ Groupe du Conseil de Recherches Medicales en Science des Radiations Faculté de Médecine, \\ Université de Sherbrooke, Sherbrooke, QC, Canada J1H $5 \mathrm{~N} 4$ \\ E-mail: lsanche@courrier.usherb.ca \\ Received May 14, 1998
}

\begin{abstract}
The impact of monochromatic low-energy electrons $(4-52 \mathrm{eV})$ is found to induce desorption of metastable particles (MP) from multilayer films formed by condensing $\mathrm{CO}$ gas on a Pt(111) substrate held at $20 \mathrm{~K}$. The results are compared with metastable production from gaseous $\mathrm{CO}$. Metastable $\mathrm{CO}$ $\left(\mathrm{CO}^{*}\right)$ is identified as the major desorbed species, its desorption is discussed in terms of intramolecular to molecule-surface vibrational energy transfer. Primary electronic excitation proceeds via the states, $a^{\prime 3} \Sigma^{+}, d^{3} \Delta, e^{3} \Sigma^{-}, I^{1} \Sigma^{-}$, and $D^{1} \Delta$ which contribute to MP desorption directly or by first decaying in high vibrational levels of the $a^{3} \Pi$ configuration. The energy threshold of $8.0 \mathrm{eV}$ for $\mathrm{CO}^{*}$ desorption indicates that $\mathrm{CO}$ in low vibrational levels of the $a^{3} \Pi$ state does not desorb. Electronic excitation occurs either near the surface of the $\mathrm{CO}$ film or in the bulk. In the latter case, excitonic motion to the surface can be followed by $\mathrm{CO}^{*}$ desorption.
\end{abstract}

PACS: $34.80 . \mathrm{Gs}, 79.20 . \mathrm{Kz}$

\section{Introduction}

Desorption from surfaces induced by electronic transitions (i.e., DIET) is a complex process which proceeds through a variety of steps including primary excitation, evolution of electronic excitation (propagation, localization, and on-site evolution, etc.) and coupling of the electronic excitation to nuclear motion [1]. While rather detailed knowledge about DIET from rare gas solids has been obtained [1], DIET of neutrals from molecular solids is not so well documented due to the additional channels involving coupling of rovibrational internal energy to the translational energy of the desorbing particle.

It is known that molecules are commonly produced and desorbed by electronic excitation on grains in interstellar media as well as on comet [2]. $\mathrm{CO}$ is a common molecule in the interstellar medium and a significant component in stellar and planetary atmospheres and comets [3]. $\mathrm{CO}$ is chosen here in our effort to understand DIET of cryogenic molecular solids. The selective detection of metastable particles (MP) provides a possibility for isolating particular processes. We report high-resolution excitation functions and time-of-flight (TOF) distributions of MP desorbed from low-temperature $\mathrm{CO}$ films by electron impact, with comparison to metastable production of gaseous $\mathrm{CO}$, in order to determine the initial electronic excitation, the desorption species and their kinetic energy. It is suggested that several vibrational excited $\mathrm{CO}^{*}$ states contribute to the measured MP signal through intramolecular to molecule-surface vibrational energy transfer.

\section{Experiment}

The experiment was performed in an ultra-highvacuum system reaching a base pressure of $\sim 10^{-10}$ Torr. The apparatus has been described in detail previously [4]. A well-collimated low-energy $(0-52 \mathrm{eV}, \Delta E=60 \mathrm{meV})$ electron beam impinges on a Pt(111) single-crystal at $18^{\circ}$ with respect to the surface normal; the desorbed charged particles are repelled by concentric grids, whereas UV photons and desorbed MP passing through the grids are measured with a large area micro-channel plate array superimposed on a position-sensitive anode. The electronic energy threshold for the MP detection is estimated to lie slightly below $6 \mathrm{eV}$, from the fact that $\mathrm{N}_{2}^{*}$ in the $A^{3} \Sigma_{u}^{+}$and $\mathrm{CO}^{*}$ in the a $a^{3} \Pi$ states 
can be detected by the micro-channel plates [4,5]. The energy of the vacuum level is calibrated $\pm 0.3 \mathrm{eV}$ by measuring the onset of the target current as the voltage between the electron source and the target is slowly increased. The crystal, which is mounted on the tip of a closed-cycle helium cryostat, can be cooled to $20 \mathrm{~K}$ and cleaned by electrical heating and Ar bombardment. The target films are grown on the Pt(111) surface by dosing CO gas with a purity of $99.99 \%$. The CO thickness is determined with an uncertainty of $\pm 50 \%$ and a reproductivity of $\pm 10 \%$ [4].

TOF measurements are performed by switching on the electron beam on for $10 \mu$ s and subsequently recording the arrival time of those particles which trigger the microchannel plates. Emission of UV photons produces a peak at $t=0$ followed by the arrival of the MP. The path length $(d)$ between the target and the detector is $5.2 \pm 0.1 \mathrm{~cm}$. This parameter is related to the kinetic energy $E(t)$ of the metastable particles by the relation

$$
E(t)=\frac{M}{2}\left(\frac{d}{t}\right)^{2}
$$

where $M$ is the mass of the metastable-particle (28 amu for CO) and $t$ is the time of flight. From this relation, we find the resolution in translational energy

$$
\Delta E(t)=\frac{M d}{t^{2}}\left(\Delta d+\frac{d}{t} \Delta t\right) .
$$

\section{Previous work on metastable production from $\mathrm{CO}$}

Our present knowledge on metastable production from $\mathrm{CO}$ by electron impact has been obtained essentially from gas-phase experiments. A summary of these studies is given here to facilitate the interpretation and discussion of our results in the next section. Our approach can be rationalized by the general similarities between the gas phase and the solid [6,7]. Electron impact excitation of $\mathrm{CO}$ in $\mathrm{CO}$ films shows that the band positions are shifted to the red by only a few tenth $\mathrm{meV}$, and the intensity distributions in the progressions are well represented by the gas phase Franck-Condon factors [7]. This is also true of $\mathrm{CO}$ excitation in light rare gas ( $\mathrm{Ne}, \mathrm{Ar})$ matrices [6]. Lifetimes of excited $\mathrm{CO}$ states are shortened in Ne matrices due to nonradiative relaxation [6], but they are not far from the radiative lifetimes in the gas phase.

Gas phase lifetimes of some $\mathrm{CO}$ excited states are listed in Table. The lowest electronic excited state $\left(a^{3} \Pi\right)$ has a lifetime of 1 to $60 \mathrm{~ms}[8,9]$. This state has been found to provide the dominant contribution to the total cross section for production of metastable $\mathrm{CO}$ by electron impact [15]. The $X^{1} \Sigma^{+} \rightarrow a^{3} \Pi$ transition is a spin-forbidden transition and the $a^{3} \Pi$ state is excited by an electron exchange process or/and cascading from higher excited states. $a^{3} \Pi$ production has a threshold at about $6 \mathrm{eV}$, goes over a maximum located at 9 to $10 \mathrm{eV}$, and then decreases continuously with electron energy [8,15-17]. By direct observation of the Cameron Band system, Ajello reported that the maximum electron-impact cross section for $a^{3} \Pi$ at about $11 \mathrm{eV}$ has a value of $1.1 \cdot 10^{-16} \mathrm{~cm}^{2}$ [16]. The value of the peak cross section was reanalyzed and raised to $1.5 \cdot 10^{-16} \mathrm{~cm}^{2}$ [17]. Excitation of the $a^{\prime 3} \Sigma^{+}$ and $d^{3} \Delta$ states, with lifetimes of several $\mu$ s [10], as well as the $b^{3} \Sigma^{+}$state can contribute to the metastable excitation function via the $a^{3} \Pi$ state [15]; i.e., through $a^{\prime 3} \Sigma^{+} \rightarrow a^{3} \Pi$, Asundi bands, $d^{3} \Delta \rightarrow a^{3} \Pi$ triplet bands and $b^{3} \Sigma^{+} \rightarrow a^{3} \Pi$, the third positive band.

Table

Lifetimes of some $\mathrm{CO}$ excited states in the gas phase

\begin{tabular}{c|c|c|c|c|c|c}
\hline \hline $\begin{array}{c}\mathrm{CO} \\
\text { state }\end{array}$ & $a^{3} \Pi$ & $a^{3} \Sigma^{+}$ & $d^{3} \Delta$ & $e^{3} \Sigma^{-}$ & $b^{3} \Sigma^{+}$ & $\begin{array}{c}I^{1} \Sigma^{-} \text {and } \\
D^{1} \Delta\end{array}$ \\
\hline \hline $\begin{array}{c}\text { Life- } \\
\text { time }\end{array}$ & $1-60 \mathrm{~ms}^{\mathrm{a}}$ & $4-10 \mu \mathrm{s}^{\mathrm{b}}$ & $3-7 \mu \mathrm{s}^{\mathrm{b}}$ & $3-8 \mu \mathrm{s}^{\mathrm{b}}$ & $60 \mathrm{~ns}^{\mathrm{c}}$ & $\begin{array}{c}80 \text { or } 97 \mu \mathrm{s}^{\mathrm{d}} \\
\text { up to } 1 \mathrm{~s}^{\mathrm{e}}\end{array}$ \\
\hline \hline
\end{tabular}

${ }^{a}$ From Refs. 8 and $9{ }^{b}$ from Ref. $10{ }^{c}$ from Ref. 11; ${ }^{d}$ from Refs. 12 and $13{ }^{\mathrm{e}}$ from Ref. 14.

A higher lying metastable state at around $10 \mathrm{eV}$ has been observed by several investigators $[8,12,13$, 18-20]. Olmsted et al. [18], using a silver-magnesium alloy surface detector, observed a strongly rising cross section with an onset at $10.5 \mathrm{eV}$ and estimated the lifetime of this metastable states to be of the order of $100 \mu$ s. They proposed that this might be the $b^{3} \Sigma^{+}$state. Using Penning-ionization detection, Cermak [20] established the existence of two metastable states, one with an energy in the range 9.2-10.2 eV and the other with an energy $\geq 10.2 \mathrm{eV}$. Borst and Zipf [8] derived the excitation cross section of the higher lying state by subtracting from the total excitation cross section the cross section of the $a^{3} \Pi$ state, observed by Ajello [16], giving a threshold at $10.4 \mathrm{eV}$. They estimated the lifetime to be $150 \mu \mathrm{s}$, and precluded the $b^{3} \Sigma{ }^{+}$state, which has a lifetime of $60 \mathrm{~ns}$ [11]. Later, Wells et al. [12] located the threshold at $9.5 \mathrm{eV}$ and determined the state's lifetime to be $97 \mu \mathrm{s}$ at $15 \mathrm{eV}$. They also deduced an electron impact cross section of 
$3 \cdot 10^{-18} \mathrm{~cm}^{2}$ at $15 \mathrm{eV}$ and proposed the $D^{1} \Delta$ and $I^{1} \Sigma^{-}$states as possible metastable states.

Mason and Newell [13] isolated the higher lying metastable state from the influence of the $a^{3} \Pi$ state by using a channel electron multiplier having a work function of about $8 \mathrm{eV}$. The threshold excitation energy was determined to be $9.45 \mathrm{eV}$ and the lifetime was deduced to be $80 \mu$ s for incident energies between threshold and $35 \mathrm{eV}$ which slightly decreases to $70 \mu$ s at electron energies above $40 \mathrm{eV}$. Maximum cross section was evaluated to be $3 \cdot 10^{-18} \mathrm{~cm}^{2}$ at $16 \mathrm{eV}$. They assigned the MP production to direct excitation of the $I^{1} \Sigma^{-}$state.

Electron impact on $\mathrm{CO}$ can also result in the formation of metastable carbon and oxygen atoms from dissociative excitation. Wells et al. [21] observed metastable fragments composed of long-lived high-Rydberg carbon and oxygen atoms and $\left.\mathrm{O}^{5} S^{0}\right)$ atoms with kinetic energies ranging from 0 to $25 \mathrm{eV}$, for impact energies in the range of 0 to $300 \mathrm{eV}$. Eight discernible TOF peaks were observed. The lowest appearance potential was found at $20 \mathrm{eV}$. An electron-impact cross section of $2 \cdot 10^{-18} \mathrm{~cm}^{2}$ at $190 \mathrm{eV}$ was deduced for the production of metastable fragments assuming that $\mathrm{O}^{*}, \mathrm{C}^{*}$, and $\mathrm{O}\left({ }^{5} S^{0}\right)$ have equal cross sections. Barnett et al. [22] found only one peak in kinetic energy distributions for the metastable oxygen fragments from carbon monoxide. The peak kinetic energy increases from 3.75 at $40 \mathrm{eV}$ to 4.69 at $60 \mathrm{eV}$. LeClair et al. [23] detected $\mathrm{O}\left({ }^{1} S\right)$ production with a threshold at $16 \mathrm{eV}$ and a maximum cross section of $5 \cdot 10^{-19} \mathrm{~cm}^{2}$ at $100 \mathrm{eV}$.

Becker et al. [24] investigated Cameron bands luminescence from CO-doped solid Ar using synchrotron radiation and thermally stimulated luminescence. They observed weak lines at $8 \mathrm{eV}$ in the excitation spectrum which corresponds to direct photoexcitation of the matrix isolated $\mathrm{CO}$ by spinallowed singlet transitions $X^{1} \Sigma^{+} \rightarrow A^{1} \Pi$. Electronic excitation of $\mathrm{CO}$ by energy transfer from excitons of the matrix was seen between $12-14 \mathrm{eV}$. They attributed the strong increase in luminescence just above $21 \mathrm{eV}$ as due to $\mathrm{CO}^{*}\left(a^{3} \Pi\right)$ production by inelastic scattering of low-energy photoelectrons. In the same system, Bahrdt et al. [25] found a CO metastable state at about $11 \mathrm{eV}$ with a lifetime of about $15 \mathrm{~ms}$. It was assigned to a $\mathrm{CO}$ quintet state populated nonradiatively after excitation of a Rydberg state.

Thresholds for electron stimulated desorption (ESD) of neutral (ground-state + metastable) molecules from several molecular solid films, including solid CO, were reported by Rakhovskaia el al. [26].
Desorption thresholds appeared at distinct electron energies: for $\mathrm{CO}$ it was about $6 \mathrm{eV}$ and assigned to excitation of the $a^{3} \Pi$ state. ESD signals below the electronic excitation threshold were smaller by a factor of 250. The desorption mechanism is believed to be conversion of electronic to vibrational and finally to translational energy via radiationless decay. More recently, Scheuer et al. [27] and Wurm et al. [28] investigated the correlation of internal excitations and substrate-adsorbate coupling for $\mathrm{CO}$ molecules desorbed from metallic and Xe surfaces by $150 \mathrm{eV}$ electron impact. They attributed the strong yield of desorbed ground state $\mathrm{CO}$ in high vibrational states to quenching, by the metal substrate, of $\mathrm{CO}^{*}$ molecules which are dissociative with respect to both the $\mathrm{C}-\mathrm{O}$ bond and the molecule-surface bond.

Desorption of ground-state neutrals from solid $\mathrm{CO}$ also has also been investigated by excitation with energetic ions $[29,30]$; the yield was found to have a quadratic dependence on the electronic stopping power of the incident particles. Chrisey et al. [30] concluded that this dependence is intrinsic to the electronic energy conversion process in $\mathrm{CO}$, i.e., intrinsic to the energy transfer process which sets molecules in motion. However, they did not identify the molecular state involved.

Low energy electron stimulated MP desorption has been investigated in our laboratory for various condensed films [4,31-33]. In the case of CO, only the yield function of MP desorption for a 50 monolayer (ML) film has been reported between 5 and $19 \mathrm{eV}$ [32]. In this paper, we report the results of experiments performed on the thickness dependence of the MP yield within the 4-52 eV electron energy range, and those obtained from the TOF distributions recorded at different impact energies within that range.

\section{Results}

The metastable yield functions of $\mathrm{CO}$ films are shown in Fig. 1 for various $\mathrm{CO}$ thicknesses. The curves are similar in shape for film thicknesses larger than $2 \mathrm{ML}$. For all $\mathrm{CO}$ thicknesses, there is a common MP desorption threshold at $8.0 \mathrm{eV}$, and the yield increases continuously with electron energy, exhibiting a broad peak at about $16 \mathrm{eV}$. These results are in agreement with those previously reported for 5-19 eV electrons impinging on a 50-ML CO film [32].

TOF distributions of desorbed MP were measured with different incident electron energy within the 10 to $50 \mathrm{eV}$ range. Apart from the small but sharp peak at $t=0$ due to $\mathrm{UV}$ photon emission, 


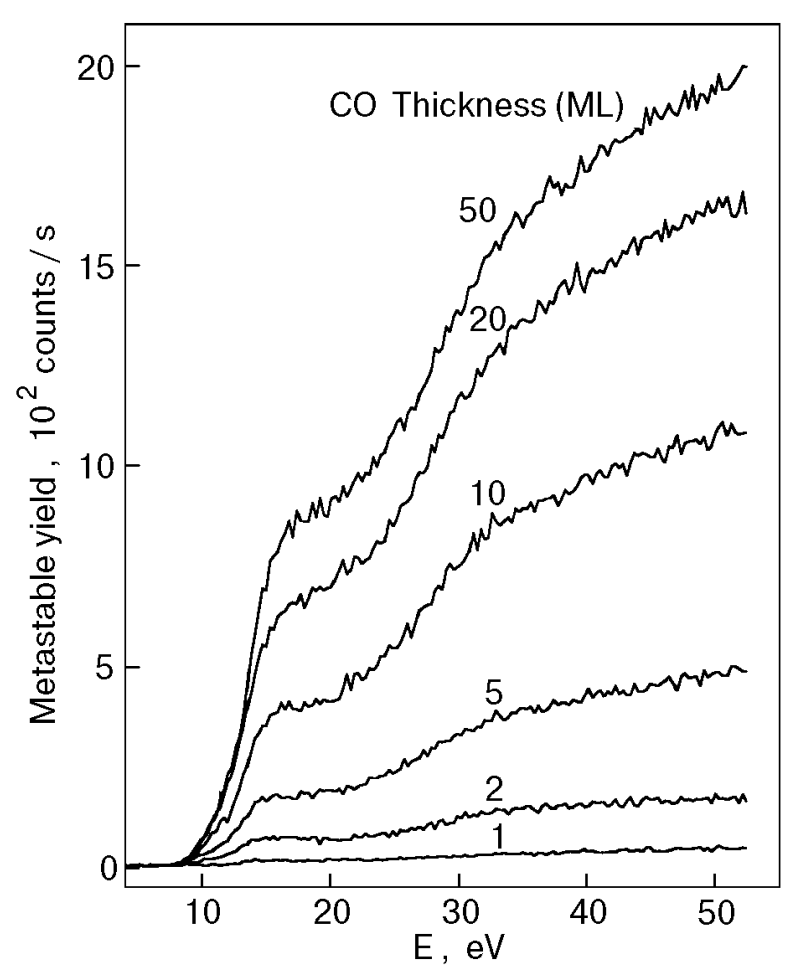

Fig. 1. Metastable carbon monoxide $\left(\mathrm{CO}^{*}\right)$ desorption yields induced by $4-52 \mathrm{eV}$ electrons impinging on $\mathrm{CO}$ films of different thicknesses indicated in monolayers (ML).

each distribution exhibits a single TOF peak; the line shape of all distribution curves are very similar to each other. In Fig. 2, TOF results are shown for a 50-ML CO film for incident energies of 11,15 and $50 \mathrm{eV}$, while the integrated peak area is plotted in the insert as a function of electron energy. The later reproduces very well the shape of the metastable yield functions recorded for thicknesses larger than

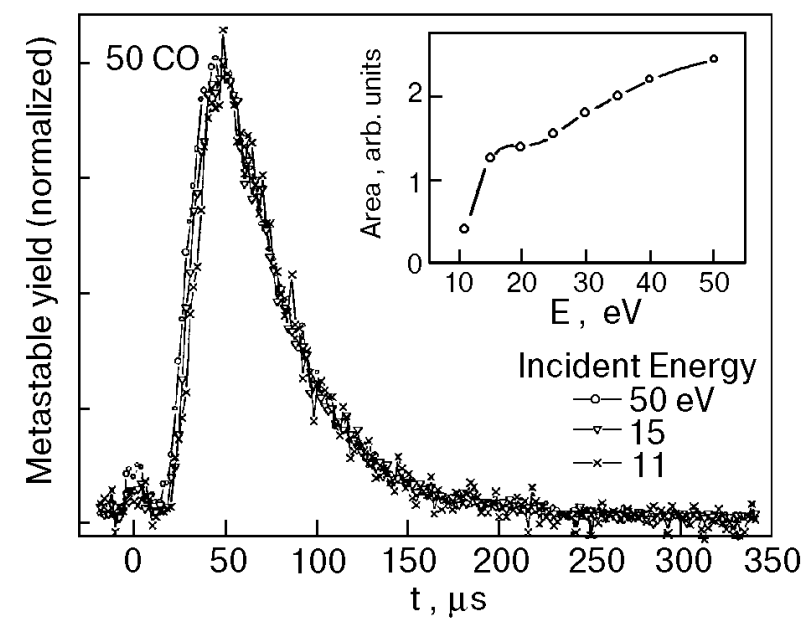

Fig. 2. Normalized time of flight (TOF) distributions of $\mathrm{CO}^{*}$ desorbed by the impact of 11-,15-, and $50-\mathrm{eV}$ electrons on a $50-$ ML CO film. The integrated TOF peak area are shown in the insert as a function of incident electron energy.

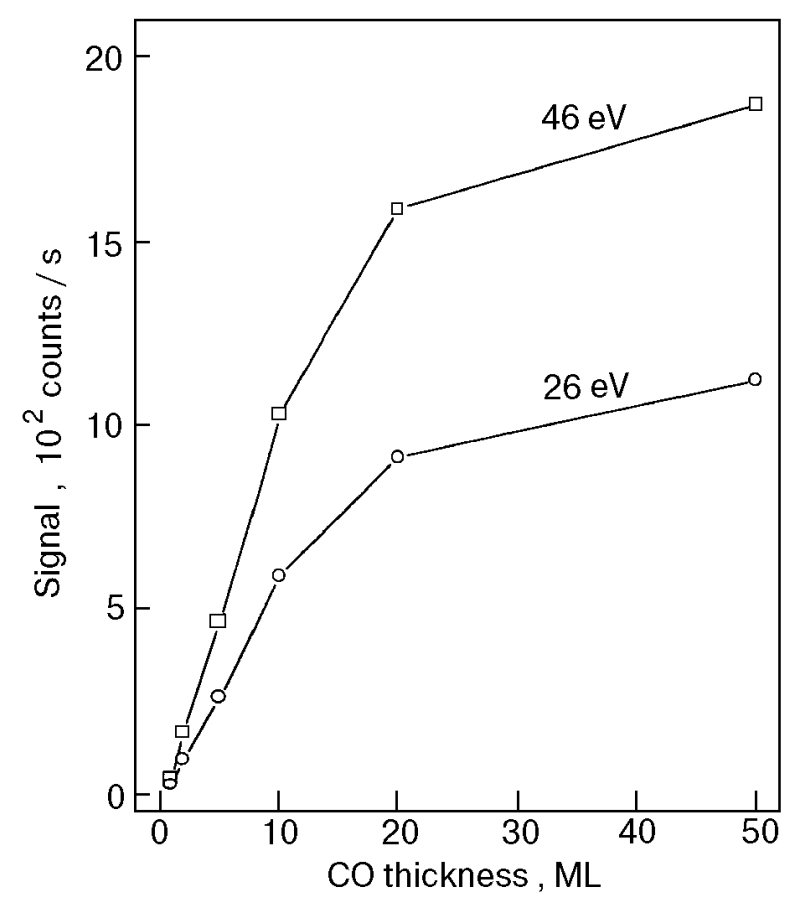

Fig. 3. $\mathrm{CO}^{*}$ signal intensity as a function of CO thickness, deduced from Fig. 1.

2 ML shown in Fig. 1. The MP distribution peak at about $45 \pm 5 \mu$ s corresponds to a kinetic energy of $195 \pm 50 \mathrm{meV}$ for $\mathrm{CO}^{*}$. With increasing electron energy, there is a slight shift in the rising slope of TOF distributions which indicates that higher energy MP are being fed into the distribution with increasing electron energy. The yield of $\mathrm{CO}^{*}$ as a function of film thickness is shown in Fig. 3 for incident electron energies of 26 and $46 \mathrm{eV}$.

\section{Discussion}

Below the electron energy of $20 \mathrm{eV}$, any detected MP signal should arise from metastable carbon monoxide $\left(\mathrm{CO}^{*}\right)$. Although LeClair et al. [23] detected an onset for the production of $\mathrm{O}\left({ }^{1} S\right)$ at $16 \mathrm{eV}$, the internal energy of this state of $4.17 \mathrm{eV}$ is too low to trigger our MP detector. The $\mathrm{O}\left({ }^{5} S^{0}\right)$ state possesses an excitation energy of $9.14 \mathrm{eV}$ and a lifetime of $180 \mu \mathrm{s}$, but it is not produced below $20 \mathrm{eV}$ [21]. The threshold energy of $8 \mathrm{eV}$ shown in Fig. 1 is higher than that of $6 \mathrm{eV}$ for the metastable $\left(a^{3} \Pi\right)$ production for gaseous CO $[8,15]$. It indicates that no significant quantities of $\mathrm{CO}$ in the $a^{3} \Pi$ state desorb from a CO film for impact energies between $6-8 \mathrm{eV}$. Thus, the threshold corresponding to $a^{3} \Pi$ excitation reported by Rakhovskaia et al. [26] in the neutral $\mathrm{CO}$ desorption yield from solid $\mathrm{CO}$ must arise essentially from $\mathrm{CO}$ molecules in their ground state. 
At low electron-impact energies, the desorption process must be simple since ionization, desorption of dissociation fragments, and desorption via collision cascade can be excluded. There are only three possibilities for the desorption of excited molecules from a molecular solid at low energies. In one case, the excited molecule at the surface has an energy higher than in the gas phase. Motion along a repulsive molecule-surface potential-energy curve can lead to acceleration and desorption of the excited molecule. This is called the cavity expulsion mechanism. In crystals with a positive electron affinity, the excited particle is not expelled owing to the attractive interaction of the excited-orbit electron cloud with the neighboring atoms. Solid CO has a positive electron affinity [34] which rules out MP desorption through cavity expulsion [35]. Another possibility is local repulsive $\mathrm{CO}^{*}-\mathrm{CO}$ interaction near the film surface which could lead to $\mathrm{CO}^{*}$ ejection in vacuum (dimeric ejection). This is impossible at low energies for $\mathrm{CO}$ because all of the valence $\mathrm{CO}$ excited states lie at lower energies in condensed $\mathrm{CO}$ than in gaseous $\mathrm{CO}$ [7]. The only possibility left is desorption via intramolecular to molecule-surface vibrational energy transfer. Direct excitation to the $a^{3} \Pi$ state only produces low intramolecular vibrational quanta $(v=0-4)$ [7] which may not allow sufficient energy transfer to the molecule-surface bond during the lifetime of the state to induce desorption [36]. This may explains the lack of $\mathrm{CO}^{*}$ desorption in the 6-8 eV range.

For a given electronic transition, the vibrational population of the $\mathrm{CO}$ molecules is determined by the Franck-Condon factors. From potential-energy diagrams of $\mathrm{CO}$ and known molecular constants [37], excitation to the $a^{3} \Sigma^{+}, d^{3} \Delta, e^{3} \Sigma^{-}, I^{1} \Sigma^{-}$, and $D^{1} \Delta$ states is expected to be in high vibrational levels. Rosenkranz and Kirby [14] calculated the Franck-Condon overlaps which peak at $v=14$ and 15 for the $I$ and $D$ states, respectively. For the $a^{\prime 3} \Sigma^{+}$state, vibrational levels $v=7-24$ lie within the Franck-Condon region, the $v=8$ level corresponds to our threshold of $8 \mathrm{eV}$ for metastable $\mathrm{CO}$ desorption observed in the present experiment. According to the near-threshold excitation spectrum of CO multilayers [7], the $a^{3} \Sigma^{+}$state is the only one produced with a non-negligible amplitude by $8-10-\mathrm{eV}$ electrons. We therefore ascribe the MP yield in the range $8-10 \mathrm{eV}$ to initial excitation to that state. Above $10 \mathrm{eV}$, the $d^{3} \Delta, e^{3} \Sigma^{-}, I^{1} \Sigma^{-}$, and $D^{1} \Delta$ states can also be excited by electron impact and therefore they are expected to be involved in MP desorption.
The $\mathrm{CO}^{*}$ molecules may desorb directly in these states or first decay into high vibrational levels of the $a^{3} \Pi$ configuration, depending on the rate of energy transferred from intramolecular vibration to the molecule-surface bond. This energy transfer is thus strongly dependent on the lifetime of the initial excited state $[38,39]$, but also on the amount of librational motion and vibrational energy of the excited molecule [36] and the surface temperature. $\mathrm{CO}$ with its high vibrational frequencies and small rotational constants has long vibrational relaxation times in the ground state, ranging from milliseconds to seconds depending on environment and other parameters [40]. Since the nonradiative contribution to the relaxation process is multiphonon [41], we may expect energy transfer to molecular-surface bond to have similar time scales. However, in the excited states, the higher vibrational energy content and the stronger molecule-surface bond may lead to shorter times for such a transfer. In fact, recent three-dimensional quantum calculations on $\mathrm{CO}$ physisorbed on a $\mathrm{NaCl}(100)$ rigid surfaces [36] show that significant desorption of the molecule can occur within picosecond times for initial librational quanta as small as 2, provided translational motion due to temperature is taken into account. Unfortunately, no such calculation exists for the case of $\mathrm{CO}$ on $\mathrm{CO}$ at cryogenic temperatures which would allow to determine if the lifetimes given in Table are sufficient for desorption of $\mathrm{CO}^{*}$ before decay to the $a^{3} \Pi$ state, when all the factors previously mentioned are taken into account. Although it is well established that $\mathrm{CO}$ desorption can occur as the result of energy transfer from intramolecular to molecule surface desorption modes, we cannot with present experimental and theoretical evidence determine the state of desorption of $\mathrm{CO}^{*}$. We therefore limit our discussion to the configurations which are initially excited by electron impact. In any case, $\mathrm{CO}^{*}$ is likely to reach our detector in the $a^{3} \Pi$ configuration. The other triplet states, having lifetimes of several $\mu$ s [10], can be radiatively de-excited into the low-lying $a^{3} \Pi$ state during the TOF. The lifetimes for $I^{1} \Sigma^{-}$and $D^{1} \Delta$ states were measured to be 80 or $97 \mu$ s, respectively [12,13], but the lifetimes of the low-lying vibrational levels of these states are very long (up to $1 \mathrm{~s}$ ) due to the small transition energies to the $A^{1} \Pi$ state [14]. Since the desorption by vibrational energy transfer lowers the vibrational levels of desorbed $\mathrm{CO}^{*}$, it may be possible that the desorbed metastables in $I$ and $D$ states live sufficiently long (presumably several hundred $\mu \mathrm{s})$ to arrive at the detector. 
Using the R-matrix method, Morgan and Tennyson [42] have calculated electron-impact excitation cross sections for the lowest seven electronically excited states of $\mathrm{CO}$ in the energy range $6-18 \mathrm{eV}$. A sharp electron resonance feature $\left({ }^{2} \Pi\right)$ is found at $10.4 \mathrm{eV}$ for the $a^{\prime 3} \Sigma^{+}$configuration, and the $d^{3} \Delta$ results show a shoulder at about $11 \mathrm{eV}$ due to a ${ }^{2} \Phi$ resonance [42]. For energy above $12 \mathrm{eV}$, the cross sections for the $a^{\prime 3} \Sigma^{+}, d^{3} \Delta$, and $e^{3} \Sigma^{-}$states are in the same order of magnitude while for the $I^{1} \Sigma^{-}$and $D^{1} \Delta$ states, they are about 5 times smaller. Interestingly, they have compared the excitation of the higher metastables $I^{1} \Sigma^{-}$and $D^{1} \Delta$ states with the measurement of Mason and Newell [13] and found roughly the same shape, with a maximum located near $16 \mathrm{eV}$. Cross sections for the excitation of all metastables, i.e., the $a, a^{\prime}, d, e, I$, and $D$ states, exhibits a maximum at $9 \mathrm{eV}$ due to the major contribution from the $a^{3} \Pi$ state and a second maximum at about $15.5 \mathrm{eV}$ [42]. The first maximum is not expected to be observed in our experiments since it is dominated by direct excitation of the $a^{3} \Pi$ state which does not directly contribute to MP desorption from $\mathrm{CO}$ films. However, considering that the near-threshold electron-impact excitation spectrum of $\mathrm{CO}$ is similar in both phases [7], we expect the energy dependence sum of the calculated cross sections of the $a^{\prime 3} \Sigma^{+}, d^{3} \Delta, e^{3} \Sigma^{-}, I^{1} \Sigma^{-}$, and $D^{1} \Delta$ states to have roughly the same shape (except the resonance features for the $a^{\prime 3} \Sigma^{+}$and $d^{3} \Delta$ states) as that of the MP yield function for $\mathrm{CO}$ films below $20 \mathrm{eV}$. We therefore ascribe the broad peak at $16 \mathrm{eV}$ in Fig. 1 to contributions from initial excitation of these states. Above $16 \mathrm{eV}, \mathrm{MP}$ yield from a $\mathrm{CO}$ film increases further while it decreases for gaseous $\mathrm{CO}$. This difference for solid and gas phase excitation possibly arises from multiple inelastic scattering of electrons in solids.

There is a slight shift in the TOF distributions in Fig. 2 with increasing electron energy. This is not expected from a $\mathrm{CO}^{*}$ signal originating from a single electronically excited state. Thus, the MP signal is believed to arise from an admixture of $\mathrm{CO}^{*}$ configurations whose relative contribution changes slightly with electron energy, assuming that the $\mathrm{MP}$ yield at $50 \mathrm{eV}$ is still dominated by molecular desorption. This is in agreement with our discussion that the states $a^{\prime 3} \Sigma^{+}, d^{3} \Delta, e^{3} \Sigma^{-}, I^{1} \Sigma^{-}$, and $D^{1} \Delta$ are all possibly involved.

Above $20 \mathrm{eV}$, metastable carbon and oxygen atoms are observed in $\mathrm{CO}$ excitation in the gas phase [21-23]. If produced in highly excited states (e.g., oxygen in the ${ }^{5} S^{0}$ state) these fragments could be detected in our experiments. Since they are created with eV's of kinetic energy they are expected to produce a signal between 8-15 $\mu$ s (4$1 \mathrm{eV}$ ) in the TOF distributions, a time domain where the magnitude of the MP yield is minimal. Hence, we suggest that highly excited atoms do not contribute significantly to the MP yields from condensed CO (i.e., the MP signal, produced by incident energy up to $50 \mathrm{eV}$, is essentially due to $\mathrm{CO}^{*}$ desorption). In the gas phase, however, the cross section for the production of atomic MP is of the order of $10^{-18} \mathrm{~cm}^{2}$, which is comparable to that of the production of $\mathrm{CO}^{*}$ in $I$ and $D$ states $[12,13$, 21-23]. The fact that we do not observe noticeable atomic MP desorption may be caused by preferential deexcitation of highly excited $\mathrm{C}$ and/or $\mathrm{O}$ atoms in the bulk and near the surface or deexcitation of dissociative states to bound states before complete separation of the $\mathrm{C}$ and $\mathrm{O}$ atoms, as suggested by Scheuer et al. [27]. Deexcitation of metastable atoms can occur by recombination, by excitation energy transfer between the atomic MP and the $\mathrm{CO}$ molecules as well as other radiationless decay and radiative transitions. Some of these processes may involve kinetic energy that induce collision cascade sputtering of neutral (ground-state) $\mathrm{CO}$ or desorption of ground state carbon and oxygen atoms, which we cannot detect.

On the other hand, molecular $\mathrm{CO}^{*}$ produced in the bulk is expected to contribute to the MP desorption by exciton motion. Analysis of the data in Fig. 1 indicates that, within the $10-52 \mathrm{eV}$ range, the MP signal increases more or less linearly with CO thickness between 2 and $10 \mathrm{ML}$; above $10 \mathrm{ML}$, the rate of increase reduces with a tendency toward saturation above $20 \mathrm{ML}$. This behavior is shown in Fig. 3 for incident energies of 26 and $46 \mathrm{eV}$.

Using the mathematical expression developed to explain the dynamics of MP desorption from $\mathrm{N}_{2}$ films [33], we obtain for a given incident electron energy the following expression for the $\mathrm{CO}^{*}$ yield $\left(Y_{d}\right)$ of a film of thickness $T$ (expressed in ML):

$$
Y_{d} \propto P_{d} \sum_{L=1}^{T}\left\{P_{\mathrm{CO}^{*}}\left[M, I_{e}(L)\right] f(L)\right\}
$$

where $P_{d}$ is the desorption probability of $\mathrm{CO}^{*}$ at the surface during a given time interval; $P_{\mathrm{CO}}{ }^{*}$ is the probability to form $\mathrm{CO}^{*}$, which is a function of the electronic excitation matrix elements $M$ and electron beam intensity $I_{e}$ at a distance $L$ from the surface; $f(L)$ is the exciton-motion function which reflects the probability of an exciton to move to the surface. Comparing the results of Fig. 3 to Eq. (3), we see that $Y_{d}$ increases with $T$, indicating a contri- 
bution to the MP yield from $\mathrm{CO}$ excitation in the bulk. $f(L)$ is necessarily non-zero for $\mathrm{CO}$ thicknesses up to $50 \mathrm{ML}$. Furthermore, these results can be interpreted as due to a $\mathrm{CO}^{*}$ yield directly proportional to the summation of CO layers in Eq. (3), with $P_{\mathrm{CO}^{*}}$ and $f(L)$ being independent of thickness in the range $2 \leq T \leq 10 \mathrm{ML}$. The slower increase above $10 \mathrm{ML}$ is probably the results of electronbeam attenuation, which reduces $P_{\mathrm{CO}^{*}}$, and /or a reduction of $f(L)$.

\section{Conclusion}

Low-energy electron stimulated desorption of metastable particles from $\mathrm{CO}$ multilayer films exhibits a threshold at $8 \mathrm{eV}$ which suggests direct excitation of the $a^{\prime 3} \Sigma^{+}$state followed by desorption in either the $a^{\prime}$ or $a^{3} \Pi$ state via intramolecular to molecular surface vibrational energy transfer. Similarly, the states $d^{3} \Delta, e^{3} \Sigma^{-}, I^{1} \Sigma^{-}$, and $D^{1} \Delta$, are believed to be the first excited and contribute to the MP signal by the same energy-transfer mechanism. In this case, desorption may also be preceded by decay into high vibrational levels of the $a^{3} \Pi$ state. Excitation of $\mathrm{CO}^{*}$ in the bulk also results in MP desorption through exciton motion. Desorption of metastable atoms in highly excited states is negligible in the electron energy range $0-52 \mathrm{eV}$. Finally, we note that, besides their interest to DIET processes, investigations such as the present one, may also be of value in the interpretation of luminescence data generated by photons [43] or charged particles [44] of sufficient energy to produce lowenergy electrons.

This work was sponsored by the Medical Research Council of Canada.

1. P. Feulner and D. Menzel, in: Laser Spectroscopy and Photochemistry on Metal Surfaces, H. -L. Dai and W. Ho (eds.), World Scientific, Singapore (1995).

2. R. E. Johnson, in: Desorption Induced by Electronic Transitions, DIET III, R. H. Stulen and M. L. Knotek (eds.), Springer, Berlin (1988), p. 47; R. E. Johnson, Energetic Charged-Particle Interactions with Atmosphere and Surfaces, Springer, Berlin (1990), p. 1 .

3. E. F. van Dishoeck and J. H. Black, Astrophys. J. 334, 771 (1988); D. L. Cooper and K. Kirby, J. Chem. Phys. 87, 424 (1987)

4. G. Leclerc, A. D. Bass, A. Mann, and L. Sanche, Phys. Rev. B46, 4865 (1992).

5. R. T. Jongma, T. Rasing, and G. Meijer, J. Chem. Phys. 102, 1925 (1995).

6. J. Bahrdt, P. Gürtler, and N. Schwentner, J. Chem. Phys. 86, 6108 (1987); J. Bahrdt and N. Schwentner, ibid. 88, 2869 (1988).

7. R. M. Marsolais, M. Michaud, and L. Sanche, Phys. Rev. A35, 607 (1987)

8. W. L. Borst and E. C. Zipf, Phys. Rev. A3, 979 (1971).
9. C. E. Johnson and R. S. van Dyck, Jr., J. Chem. Phys. 56, 1506 (1972)

10. H. A. van Sprang, G. R. Möhlmann, and F. J. de Heer, Chem. Phys. 24, 429 (1977); S. Shadfar, S. R. Lorentz, W. C. Paske, and D. E. Golden, J. Chem. Phys. 76, 5838 (1982); W. C. Paske, A. W. Garrett, S. Shadfar, D. E. Golden, and J. R. Twist, ibid. 76, 3002 (1982).

11. R. Anderson, Atomic Data 3, 227 (1971); A. J. Smith, R. E. Imhof, and F. H. Read, J. Phys. B6, 1333 (1973).

12. W. C. Wells, W. L. Borst, and E. C. Zipf, Phys. Rev. A8 2463 (1973)

13. N. J. Mason and W. R. Newell, J. Phys. B21, 1293 (1988).

14. M. E. Rosenkranz and K. Kirby, J. Chem. Phys. 90, 6528 (1989).

15. D. S. Newman, M. Zubek, and G. C. King, J. Phys. B16, 2247 (1983)

16. J. M. Ajello, J. Chem. Phys. 55, 3158 (1971).

17. P. W. Erdman and E. C. Zipf, Planet. Space Sci. 31, 317 (1983); J. M. Furlong and W. R. Newell, J. Phys. B29, 331 (1996).

18. J. Olmsted III, A. S. Newton, and K. Street, Jr., J. Chem. Phys. 42, 2321 (1965).

19. J. N. H. Brunt, G. C. King, and F. H. Read, J. Phys. B11 173 (1978)

20. V. Cermak, J. Chem. Phys. 44, 1318 (1966).

21. W. C. Wells, W. L. Borst, and E. C. Zipf, Phys. Rev. A17, 1357 (1978).

22. S. M. Barnett, N. J. Mason, and Newell, J. Phys. B25, 1307 (1992).

23. L. R. LeClair, M. D. Brown, and J. W. McConkey, Chem. Phys. 189, 769 (1994).

24. J. Becker, O. N. Grigorashchenko, A. N. Ogurtsov, M. Runne, E.V. Savchenko, and G. Zimmerer, J. Phys. D31, 749 (1998)

25. J. Bahrdt and N. Schwentner, Chem. Phys. 127, 263 (1988); J. Bahrdt, H. Nahme, and N. Schwentner, ibid. 144, 273 (1990)

26. O. Rakhovskaia, P. Wiethoff, and P. Feulner, Nucl. Instrum. Methods Phys. Res. B101, 169 (1995).

27. M. Scheuer, D. Menzel, and P. Feulner, Surf Sci. 390, 23 (1997).

28. S. Wurm, P. Feulner, and D. Menzel, Phys. Rev. Lett. 74 2591 (1995).

29. W. L. Brown, W. M. Augustyniak, K. J. Marcantonio, E. H. Simmons, J. W. Boring, R. E. Johnson, and C. T. Reimann, Nucl. Instrum. Methods B1, 307 (1984); J. Schou, O. Ellegaard, P. Børgesen, and H. Sørensen, in: Desorption Induced by Electronic Transitions, DIET II, W. Brenig and D. Menzel (eds.), Springer, Berlin (1985), p. 170.

30. D. B. Chrisey, W. L. Brown, and J. W. Boring, Surf. Sci. 225, 130 (1990).

31. G. Leclerc, A. D. Bass, M. Michaud, and L. Sanche, $J$. Electron Spectrosc. Relat. Phenom. 52, 725 (1990); A. Mann, G. Leclerc, and L. Sanche, Phys. Rev. B46, 9683 (1992); A. Mann, P. Cloutier, D. Liu, and L. Sanche, ibid. 51, 7200 (1995)

32. H. Shi, P. Cloutier, and L. Sanche, Phys. Rev. B52, 5385 (1995).

33. H. Shi, P. Cloutier, J. Gamache, and L. Sanche, Phys. Rev. B53, 13830 (1995).

34. G. Bader, G. Perluzzo, L. G. Caron, and L. Sanche, Phys Rev. B30, 78 (1984).

35. F. Coletti, J. M. Debever, and G. Zimmerer, J. Phys. (Paris) Lett. 45, 467 (1984). 
36. F. Dzegilenko, E. Herbst, and T. Uzer, J. Chem. Phys. 102, 2593 (1995) and citations therein; E. Galloway and E. Herbst, Astron. Astrophys. 287, 633 (1994).

37. K. P. Huber and G. Herzberg, Constants of Diatomic Molecules, Van Nostrand Reinhold, New York (1979); S. G. Tilford and J. D. Simmons, J. Phys. Chem. Ref. Data 1, 147 (1972).

38. H. J. Kreuzer and D. N. Lowy, Chem. Phys. Lett. 78, 50 (1981); Z. W. Gortel, H. J. Kreuzer, P. Piercy, and R. Teshima, Phys. Rev. B27, 5066 (1983); 28, 2119 (1983); H. J. Kreuzer and Z. W. Gortel, ibid. 29, 6926 (1984).
39. B. Fain and S. H. Lin, Chem. Phys. Lett. 114, 497 (1985); B. Fain, ibid. 118, 283 (1985).

40. H. Dubost, in: Inert Gases, M. Klein (ed.), Springer, Berlin (1984).

41. S. A. Egorov and J. L. Skinner, J. Chem. Phys. 106, 1034 (1997) and citations therein.

42. L. A. Morgan and J. Tennyson, J. Phys. B26, 2429 (1993).

43. A. N. Ogurtsov, E. V. Savchenko, J. Becker, M. Runne, and G. Zimmerer, Low Temp. Phys. 23, 851 (1997).

44. See for example, E. V. Savchenko, A. N. Ogurtsov, O. N. Grigorashchenko, and S. A. Gubin, Chem. Phys. 189, 415 (1994). 